TRANSACTIONS OF THE

AMERICAN MATHEMATICAL SOCIETY

Volume 352, Number 11, Pages 5045-5062

S 0002-9947(00)02635-0

Article electronically published on July 18, 2000

\title{
THE DENSITY OF RATIONAL LINES ON CUBIC HYPERSURFACES
}

\author{
SCOTT T. PARSELL
}

\begin{abstract}
We provide a lower bound for the density of rational lines on the hypersurface defined by an additive cubic equation in at least 57 variables. In the process, we obtain a result on the paucity of non-trivial solutions to an associated system of Diophantine equations.
\end{abstract}

\section{INTRODUCTION}

The existence of linear spaces on algebraic varieties was first investigated by Brauer 4] and Birch [3] in the middle part of the 20th century, but the analysis of the density of such spaces has begun only very recently. The author [10] has shown that if $c_{1}, \ldots, c_{s}$ are non-zero integers, then the hypersurface defined by the additive equation

$$
c_{1} z_{1}^{k}+\cdots+c_{s} z_{s}^{k}=0
$$

contains the expected density of rational lines, provided that

$$
s \geq\left(\frac{14}{3}+o(1)\right) k^{2} \log k
$$

and that the obvious local solubility requirements are met. The purpose of the present note is to obtain an explicit upper bound for the number of variables required in the case $k=3$.

Given an affine line $\ell: \mathbf{x} t+\mathbf{y}$, we define its "height" by $h(\ell)=\max \left(\left|x_{i}\right|,\left|y_{i}\right|\right)$. Further, when $c_{1}, \ldots, c_{s}$ are non-zero integers, we write $L_{s}(P)$ for the number of distinct rational lines $\ell$ having $h(\ell) \leq P$ and lying on the hypersurface defined by

$$
c_{1} z_{1}^{3}+\cdots+c_{s} z_{s}^{3}=0 \text {. }
$$

Our main result is the following.

Theorem 1.1. Suppose that $s \geq 57$. Then one has

$$
L_{s}(P) \gg P^{2 s-12}
$$

for P sufficiently large.

For comparison, we note that Wooley [19] has demonstrated the existence of rational lines on arbitrary cubic hypersurfaces in at least 37 variables, whereas we require 57 variables in Theorem 1.1. In the additive situation we are considering, the existence of lines follows immediately from the theory of a single additive cubic

Received by the editors May 21, 1999 and, in revised form, July 23, 1999.

2000 Mathematics Subject Classification. Primary 11D25, 11D45, 11L03, 11P55.

Research supported in part by NSF grant DMS-9622773 and by a fellowship from the David and Lucile Packard Foundation. 
equation (see R. Baker [2]), provided that $s \geq 14$, and hence the significance of our result lies in the density estimate.

The existence of these "trivial" lines when $s \geq 14$ is in fact key to our analysis, for they give rise to non-singular integer solutions of the system

$$
c_{1} x_{1}^{3-j} y_{1}^{j}+\cdots+c_{s} x_{s}^{3-j} y_{s}^{j}=0 \quad(0 \leq j \leq 3)
$$

and hence allow us to avoid imposing explicit local solubility hypotheses in Theorem 1.1. Unfortunately, the solutions arising in this way are singular for larger values of $k$ and are therefore of no use in the analysis of the more general problem considered in 10. Local conditions also may present an obstacle to demonstrating the expected density of higher-dimensional linear spaces on a cubic hypersurface in a reasonable number of variables. While the results of Schmidt [11, [12] could be applied to the analogues of (1.1), the number of variables required may in general be quite large.

We prove Theorem 1.1 by applying the Hardy-Littlewood method to count the number of solutions of (1.1) lying in a given box. In order to obtain estimates for mean values of the underlying exponential sums, we will be required to investigate certain auxiliary systems of equations. For example, let $S(P)$ denote the number of solutions of the system

$$
\begin{aligned}
x_{1}^{3}+x_{2}^{3}+x_{3}^{3} & =x_{4}^{3}+x_{5}^{3}+x_{6}^{3}, \\
x_{1}^{2} y_{1}+x_{2}^{2} y_{2}+x_{3}^{2} y_{3} & =x_{4}^{2} y_{4}+x_{5}^{2} y_{5}+x_{6}^{2} y_{6}, \\
x_{1} y_{1}^{2}+x_{2} y_{2}^{2}+x_{3} y_{3}^{2} & =x_{4} y_{4}^{2}+x_{5} y_{5}^{2}+x_{6} y_{6}^{2}, \\
y_{1}^{3}+y_{2}^{3}+y_{3}^{3} & =y_{4}^{3}+y_{5}^{3}+y_{6}^{3},
\end{aligned}
$$

with $x_{i}, y_{i} \in[1, P] \cap \mathbb{Z}$. Further, write $T(P)$ for the number of "trivial" solutions, in which

$$
\left(x_{4}, x_{5}, x_{6}\right)=\sigma\left(x_{1}, x_{2}, x_{3}\right) \quad \text { and } \quad\left(y_{4}, y_{5}, y_{6}\right)=\sigma\left(y_{1}, y_{2}, y_{3}\right)
$$

for some permutation $\sigma \in S_{3}$. The following "paucity" result shows that almost all solutions counted by $S(P)$ are of this diagonal type.

Theorem 1.2. For every $\varepsilon>0$, one has

$$
S(P)-T(P) \ll_{\varepsilon} P^{6-\frac{11}{192}+\varepsilon} .
$$

Clearly, one has $T(P)=6 P^{6}+O\left(P^{4}\right)$, and it follows that $S(P) \sim 6 P^{6}$. In Section 3 , we interpret this diagonal behavior as giving a best-possible estimate for the 6 th moment of the exponential sum

$$
F(\boldsymbol{\alpha})=\sum_{1 \leq x, y \leq P} e\left(\alpha_{0} x^{3}+\alpha_{1} x^{2} y+\alpha_{2} x y^{2}+\alpha_{3} y^{3}\right)
$$

and then use the iterative method developed in [10] to obtain non-trivial estimates for higher moments. After deriving some simple Weyl estimates in Section 4, we are able to complete the proof of Theorem 1.1 in Section 5 by using the HardyLittlewood method as in [10, $\S 10$.

Our methods apply equally well to a generalization of Waring's problem in which we seek to represent a polynomial of degree $k$ as a sum of $k$ th powers of linear polynomials. This application was discussed in detail in [10], where the function $G_{1}^{*}(k)$ was introduced to denote the number of $k$ th powers required to represent all polynomials whose coefficients are sufficiently large and compatible with all local solubility considerations. The bound $G_{1}^{*}(3) \leq 55$ is immediate on combining the 
analysis of [10], $\S 9$, with that of the present paper, so we feel justified in omitting the details.

The author gratefully acknowledges the many helpful suggestions offered by Professor Trevor Wooley during the writing of the paper. This work would not have been possible without his keen insight and constant encouragement. The author also thanks the referee for useful comments.

\section{The Paucity Problem}

Our goal in this section is to establish Theorem 1.2 Before proceeding with the proof, we record for reference some of the key estimates we will use. The first of these is implicit in the work of Hooley [7] on sums of four cubes.

Lemma 2.1. Let $n$ be a non-zero integer, and let $R(P)$ denote the number of integral solutions of the equation

$$
x_{1}^{3}+x_{2}^{3}+x_{3}^{3}+x_{4}^{3}=n
$$

with $\left|x_{i}\right| \leq P$. Then one has $R(P) \ll P^{11 / 6+\varepsilon}$.

Proof. Clearly, we may focus attention on solutions in which at least two of the $x_{i}$ are non-zero. For any such solution $\mathbf{x}$ counted by $R(P)$, we can find $i$ and $j$ such that $x_{i}$ and $x_{j}$ have the same parity and are not both zero. Now if $x_{i}+x_{j}=0$ and $x_{k}$ and $x_{l}$ are the remaining two variables, then since $n \neq 0$ we must have $x_{k}+x_{l} \neq 0$, and if $x_{k}$ and $x_{l}$ do not have the same parity, then one of them has the same parity as $x_{i}$ and $x_{j}$. Thus, after relabeling variables, we may assume that $x_{1} \equiv x_{2}(\bmod 2)$ and $x_{1} \neq-x_{2}$. This allows us to write $x_{1}=r+s$ and $x_{2}=r-s$, where $r$ and $s$ are integers with $r \neq 0$, and hence to consider solutions of the equation

$$
2 r\left(r^{2}+3 s^{2}\right)=n-z^{3}-w^{3} .
$$

The argument is now identical to that of Hooley 7, the condition $r \neq 0$ being essential to the consideration of congruences modulo divisors of $r$. The only change is that the upper bound of $n^{1 / 3}$ for the moduli of $r, z$, and $w$ is replaced throughout by $P$, and the sieving parameter $\xi$ is now chosen to be $P^{1 / 6}$.

We also make use of some recent work of Heath-Brown [ $[6]$ on sums of two cubes.

Lemma 2.2. Let $U(P)$ denote the number of integral solutions of the equation

$$
x_{1}^{3}+x_{2}^{3}=x_{3}^{3}+x_{4}^{3}
$$

with $\left|x_{i}\right| \leq P$ and $x_{1}+x_{2} \neq x_{3}+x_{4}$. Then one has $U(P) \ll P^{4 / 3+\varepsilon}$.

Proof. This is a special case of Heath-Brown [6], Theorem 1.

We remark that Hooley [8], using the Riemann hypothesis for varieties over finite fields, obtained a result of the above shape with the exponent $4 / 3$ replaced by $5 / 3$. Wooley [18] later devised an elementary proof of this result, and his ideas play a key role in Heath-Brown's argument.

Finally, we recall a result on binary quadratic forms dating back to Estermann [5]. 
Lemma 2.3. Let $a, b$, and $c$ be non-zero integers, and let $Q(P)$ denote the number of integral solutions of the equation

$$
a x^{2}+b y^{2}=c
$$

with $1 \leq x, y \leq P$. Then one has $Q(P) \ll|a b c P|^{\varepsilon}$.

Proof. See (for example) Vaughan and Wooley [15], Lemma 3.5.

We are now ready to embark on the proof of Theorem 1.2. On writing $h=x_{1}-x_{4}$ and $g=y_{1}-y_{4}$ and relabeling variables in 1.2 , we see that $S(P)$ is the number of solutions of the system of equations

$$
\begin{aligned}
h\left(3 x(x+h)+h^{2}\right) & =u_{1}^{3}+u_{2}^{3}-u_{3}^{3}-u_{4}^{3}, \\
\left(2 h x+h^{2}\right) y+g(x+h)^{2} & =u_{1}^{2} v_{1}+u_{2}^{2} v_{2}-u_{3}^{2} v_{3}-u_{4}^{2} v_{4}, \\
\left(2 g y+g^{2}\right) x+h(y+g)^{2} & =u_{1} v_{1}^{2}+u_{2} v_{2}^{2}-u_{3} v_{3}^{2}-u_{4} v_{4}^{2}, \\
g\left(3 y(y+g)+g^{2}\right) & =v_{1}^{3}+v_{2}^{3}-v_{3}^{3}-v_{4}^{3},
\end{aligned}
$$

with

$$
1 \leq x, y, u_{i}, v_{i} \leq P \quad \text { and } \quad|h|,|g|<P .
$$

We shall estimate $N(P)=S(P)-T(P)$ by dividing into several cases.

(i) Let $N_{1}$ denote the number of solutions counted by $N(P)$ for which $h=g=0$, and consider a solution $x, y, \mathbf{u}, \mathbf{v}$ counted by $N_{1}$. Then one has

$$
\left(u_{1}, u_{2}, v_{1}, v_{2}\right) \neq\left(u_{3}, u_{4}, v_{3}, v_{4}\right) \quad \text { and } \quad\left(u_{1}, u_{2}, v_{1}, v_{2}\right) \neq\left(u_{4}, u_{3}, v_{4}, v_{3}\right) \text {, }
$$

since otherwise the solution would be counted by $T(P)$. If we have $\left(u_{1}, u_{2}\right)=$ $\left(u_{3}, u_{4}\right)$ and $\left(v_{1}, v_{2}\right)=\left(v_{4}, v_{3}\right)$, then the second equation in 2.1) implies that either $u_{1}=u_{2}$ or $v_{1}=v_{2}$, whence the number of choices for $\mathbf{u}$ and $\mathbf{v}$ is $O\left(P^{3}\right)$. Trivially, there are $O\left(P^{2}\right)$ choices for $x$ and $y$, so the total number of solutions is $O\left(P^{5}\right)$, and the same analysis applies if $\left(u_{1}, u_{2}\right)=\left(u_{4}, u_{3}\right)$ and $\left(v_{1}, v_{2}\right)=\left(v_{3}, v_{4}\right)$. Otherwise, since $u_{i}$ and $v_{i}$ are positive, it follows that either $u_{1}+u_{2} \neq u_{3}+u_{4}$ or $v_{1}+v_{2} \neq v_{3}+v_{4}$, so Lemma 2.2 may be applied to estimate the number of choices for $\mathbf{u}$ or $\mathbf{v}$ (or possibly both). On combining this with Hua's Lemma, one sees that $N_{1} \ll P^{16 / 3+\varepsilon}$.

(ii) Let $N_{2}$ denote the number of solutions counted by $N(P)$ for which exactly one of $h$ or $g$ is zero. Suppose first that $h=0$ and $g \neq 0$. Then by Hua's Lemma one has $O\left(P^{2+\varepsilon}\right)$ choices for $\mathbf{u}$, and by a trivial estimate there are $O\left(P^{2}\right)$ choices for $g$ and $y$. Now for fixed non-zero $g$ and $y$, we may apply Lemma 2.1 to deduce that there are $O\left(P^{11 / 6+\varepsilon}\right)$ choices of $\mathbf{v}$ satisfying the fourth equation of (2.1). Finally, since $g \neq 0$, the second equation is a non-trivial polynomial in $x$ and hence determines $x$ to $O(1)$. By following a symmetric argument in the case where $g=0$ and $h \neq 0$, we find that $N_{2} \ll P^{35 / 6+\varepsilon}$.

(iii) Write $d=(h, g)$, let $\beta$ be a parameter at our disposal, and let $N_{3}$ denote the number of solutions counted by $N(P)$ for which $h g \neq 0$ and $|h g / d| \leq P^{1+\beta}$. In this case, there are $O\left(P^{1+\beta}\right)$ choices for the integer $h g / d$, of which $d, h / d$ and $g / d$ are all divisors. Thus by a standard estimate for the divisor function, we see that there are $O\left(P^{1+\beta+\varepsilon}\right)$ choices for $h$ and $g$. Trivially, there are $O(P)$ choices for $x$, and then by Lemma 2.1 we have $O\left(P^{11 / 6+\varepsilon}\right)$ choices for $\mathbf{u}$. Now by taking a 
linear combination of the equations (2.1), with respective weights $g^{3},-3 g^{2} h, 3 g h^{2}$, and $-h^{3}$, we find that any solution $x, y, g, h, \mathbf{u}, \mathbf{v}$ satisfies

$$
\left(g u_{1}-h v_{1}\right)^{3}+\left(g u_{2}-h v_{2}\right)^{3}=\left(g u_{3}-h v_{3}\right)^{3}+\left(g u_{4}-h v_{4}\right)^{3},
$$

and by applying Hua [9], Theorem 4, to the underlying mean value we find that, for fixed $h, g$, and $\mathbf{u}$, there are $O\left(P^{2+\varepsilon}\right)$ choices for $\mathbf{v}$. Finally, $y$ is determined to $O(1)$ by a polynomial, whence $N_{3} \ll P^{35 / 6+\beta+\varepsilon}$.

(iv) For $i=1, \ldots, 4$, write $X_{i}=g u_{i}-h v_{i}$, and let $N_{4}$ denote the number of solutions counted by $N(P)$ for which $X_{1}+X_{2}=X_{3}+X_{4}$ and $h g \neq 0$. The former condition, when combined with (2.3), implies that either $X_{1}=X_{3}, X_{2}=X_{3}$, or $X_{1}=-X_{2}$. We may suppose that $X_{1}=X_{3}$ and $X_{2}=X_{4}$, so that

$$
g\left(u_{1}-u_{3}\right)=h\left(v_{1}-v_{3}\right) \text { and } g\left(u_{2}-u_{4}\right)=h\left(v_{2}-v_{4}\right),
$$

the argument in the remaining two cases being identical. For convenience we write

$$
A=u_{1}-u_{3}, \quad B=u_{2}-u_{4}, \quad C=v_{1}-v_{3}, \quad \text { and } \quad D=v_{2}-v_{4} .
$$

Since $h$ and $g$ are non-zero, the first equation in (2.1) implies that either $A$ or $B$ is non-zero, and the fourth equation implies that either $C$ or $D$ is non-zero. Suppose that $C \neq 0$ and $D=0$. We first choose $u_{2}=u_{4}$ and $v_{2}=v_{4}$ in $O\left(P^{2}\right)$ ways, and then by (2.1) we have

$$
(x+h)^{3}+u_{3}^{3}=x^{3}+u_{1}^{3} \quad \text { and } \quad(y+g)^{3}+v_{3}^{3}=y^{3}+v_{1}^{3} .
$$

Since solutions with $x=u_{3}$ and $y=v_{3}$ are counted by $T(P)$, we may apply Lemma 2.2, together with Hua's Lemma, to deduce that there are $O\left(P^{10 / 3+\varepsilon}\right)$ choices for $x, y, h, g, u_{1}, u_{3}, v_{1}$, and $v_{3}$. The case where $C=0$ and $D \neq 0$ is identical.

It remains to consider solutions for which both $C$ and $D$ (and hence $A$ and $B$ ) are non-zero. We first observe that, after substituting from (2.5) and completing the square, the first and fourth equations in (2.1) become

$$
h\left(3 x(x+h)+h^{2}\right)-\frac{1}{4}\left(A^{3}+B^{3}\right)=3 A\left(u_{3}+\frac{1}{2} A\right)^{2}+3 B\left(u_{4}+\frac{1}{2} B\right)^{2}
$$

and

$$
g\left(3 y(y+g)+g^{2}\right)-\frac{1}{4}\left(C^{3}+D^{3}\right)=3 C\left(v_{3}+\frac{1}{2} C\right)^{2}+3 D\left(v_{4}+\frac{1}{2} D\right)^{2},
$$

respectively. In view of (2.6) and (2.7), we further classify solutions according to whether

$$
h\left(3 x(x+h)+h^{2}\right)-\frac{1}{4}\left(A^{3}+B^{3}\right)=0
$$

or

$$
g\left(3 y(y+g)+g^{2}\right)-\frac{1}{4}\left(C^{3}+D^{3}\right)=0 .
$$

If both (2.8) and (2.9) hold, then we start by selecting values for $A$ and $B$ from among $O\left(P^{2}\right)$ possibilities, and (2.8) then determines $h$ and $x$ to $O\left(P^{\varepsilon}\right)$. Trivially, there are $O(P)$ choices for $g$, and (2.4) then determines $C$ and $D$ to $O\left(P^{\varepsilon}\right)$, whence $y$ is determined to $O(1)$ by (2.9). Finally, $u_{3}$ and $v_{3}$ may be assigned in $O\left(P^{2}\right)$ ways, and this choice determines $\mathbf{u}$ and $\mathbf{v}$ to $O(1)$ in light of (2.5), (2.6), and (2.7). Hence there are $O\left(P^{5+\varepsilon}\right)$ solutions of this type.

If (2.8) holds but (2.9) does not, then we assign $A, B$, and $u_{3}$ in $O\left(P^{3}\right)$ ways, so that $\mathbf{u}$ is determined to $O(1)$ by (2.6). Then $h$ and $x$ are again determined up to $O\left(P^{\varepsilon}\right)$, and there are $O\left(P^{2}\right)$ choices for $y$ and $g$. This latter choice determines $C$ and $D$ to $O\left(P^{\varepsilon}\right)$ by (2.4), and we may apply Lemma 2.3) to (2.7), regarded as a binary quadratic equation in the variables $v_{3}$ and $v_{4}$. The case where (2.9) holds 
but (2.8) does not is exactly symmetric, so we see that there are $O\left(P^{5+\varepsilon}\right)$ solutions of these two types.

Finally, if neither (2.8) nor (2.9) holds, then we fix $h, C$, and $D$ in $O\left(P^{3}\right)$ ways, from which $g, A$, and $B$ are determined to $O\left(P^{\varepsilon}\right)$ by (2.4). There are $O\left(P^{2}\right)$ possibilities for $x$ and $y$, and Lemma 2.3 then shows that $\mathbf{u}$ and $\mathbf{v}$ are determined up to $O\left(P^{\varepsilon}\right)$ by (2.6) and (2.7). Thus we conclude that $N_{4} \ll P^{16 / 3+\varepsilon}$.

(v) Now let $\gamma$ be a parameter at our disposal, write $M=P^{1+\beta}$, and let $N_{5}$ be the number of solutions counted by $N(P)$ in which

$$
h g \neq 0, \quad|h g / d|>M, \quad X_{1}+X_{2} \neq X_{3}+X_{4},
$$

and $d=(h, g) \leq P^{\gamma}$. By symmetry, we may assume that $|h| \geq|g|$, the argument in the other case being identical. Write $h^{\prime}=h / d$ and $g^{\prime}=g / d$, so that $\left(h^{\prime}, g^{\prime}\right)=1$. For any given $d$ and $\left|h^{\prime}\right| \geq\left|g^{\prime}\right|$, we divide both sides of (2.3) by $d^{3}$ and apply Lemma 2.2 to deduce that there are then $O\left(\left(\left|h^{\prime}\right| P\right)^{4 / 3+\varepsilon}\right)$ possible choices for $X_{1}, \ldots, X_{4}$. With $X_{i}$ now fixed and $\left(h^{\prime}, g^{\prime}\right)=1$, any two choices for $u_{i}$ must be congruent modulo $h^{\prime}$, so one has $O\left(P /\left|h^{\prime}\right|\right)$ possibilities for each of $u_{1}, \ldots, u_{4}$, and this determines $\mathbf{v}$. Since $x$ and $y$ are then determined by polynomials, we find that

$$
\begin{aligned}
N_{5} & \ll \sum_{d \leq P^{\gamma}} \sum_{1 \leq g \leq P / d} \sum_{h \geq \max (g, M / g d)}(h P)^{4 / 3+\varepsilon}(P / h)^{4} \\
& \ll P^{16 / 3+\varepsilon} \sum_{d \leq P^{\gamma}}\left(\sum_{g \leq(M / d)^{1 / 2}} \sum_{h \geq M / g d} h^{-8 / 3}+\sum_{g>(M / d)^{1 / 2}} \sum_{h \geq g} h^{-8 / 3}\right) \\
& \ll P^{16 / 3+\varepsilon} \sum_{d \leq P^{\gamma}}\left(\sum_{g \leq(M / d)^{1 / 2}}(M / g d)^{-5 / 3}+\sum_{g>(M / d)^{1 / 2}} g^{-5 / 3}\right) \\
& \ll P^{16 / 3+\varepsilon} \sum_{d \leq P^{\gamma}}\left((M / d)^{-5 / 3}(M / d)^{4 / 3}+(M / d)^{-1 / 3}\right) \\
& \ll P^{16 / 3+\varepsilon} M^{-1 / 3} \sum_{d \leq P^{\gamma}} d^{1 / 3} \ll P^{\frac{16}{3}-\frac{1}{3}(1+\beta)+\frac{4}{3} \gamma+\varepsilon} .
\end{aligned}
$$

(vi) Finally, let $N_{6}$ be the number of solutions counted by $N(P)$ with (2.10) and $d>P^{\gamma}$. In this case we use an affine slicing approach almost exactly as in Wooley [18]. As before, we exploit the symmetry of our system to focus attention on solutions with $|h| \geq|g|$. On recalling (2.3), we have that

$$
X_{1}^{3}+X_{2}^{3}=X_{3}^{3}+X_{4}^{3} \quad \text { and } \quad X_{1}+X_{2}=X_{3}+X_{4}+H
$$

for some integer $H$. For convenience, we write $X_{i}^{\prime}=X_{i} / d$ and $H^{\prime}=H / d$. For fixed $h, g$, and $\mathbf{u}$, one has

$$
H^{\prime}=g^{\prime}\left(u_{1}+u_{2}-u_{3}-u_{4}\right)-h^{\prime}\left(v_{1}+v_{2}-v_{3}-v_{4}\right),
$$

which determines the residue class of $H^{\prime}$ modulo $h^{\prime}$. Furthermore, since $\left|h^{\prime}\right| \geq\left|g^{\prime}\right|$, one has $\left|H^{\prime}\right| \leq 4\left|h^{\prime}\right| P$. Now from the equations (2.11), we find that

$$
\left(X_{1}+X_{2}-X_{3}\right)^{3}-\left(X_{1}^{3}+X_{2}^{3}-X_{3}^{3}\right)=\left(X_{4}+H\right)^{3}-X_{4}^{3},
$$

which simplifies to

$$
3\left(X_{1}-X_{3}\right)\left(X_{2}-X_{3}\right)\left(X_{1}+X_{2}\right)=H\left(3 X_{4}^{2}+3 X_{4} H+H^{2}\right) .
$$


By (2.10), we have $H \neq 0$, so after dividing both sides of (2.12) by $d^{3}$ we see that at least one of $X_{1}^{\prime}-X_{3}^{\prime}, X_{2}^{\prime}-X_{3}^{\prime}$, or $X_{1}^{\prime}+X_{2}^{\prime}$ has a divisor $e \gg\left|H^{\prime}\right|^{1 / 3}$ in common with $H^{\prime}$. We suppose that

$$
e=\left(H^{\prime}, X_{1}^{\prime}-X_{3}^{\prime}\right) \gg\left|H^{\prime}\right|^{1 / 3}
$$

and write $X_{1}-X_{3}=d e Y$, the analysis in the other two cases being identical. Then, for fixed $d$ and $e$, there are $O\left(\left|h^{\prime}\right| P / e\right)$ choices for $Y$. Now, on substituting $X_{4}=X_{1}+X_{2}-X_{3}-H$ and $X_{3}=X_{1}-d e Y$ in (2.12), we obtain

$$
\begin{gathered}
3 \mathrm{de} Y X_{1}^{2}-3(\mathrm{de} Y-H) X_{2}^{2}-3(\mathrm{de} Y)^{2} X_{1}-3(\mathrm{de} Y-H)^{2} X_{2} \\
=(d e Y-H)^{3}-(\mathrm{de} Y)^{3},
\end{gathered}
$$

and after completing the square this becomes

$$
\begin{gathered}
3 \operatorname{de} Y\left(X_{1}-\frac{1}{2} d e Y\right)^{2}-3(d e Y-H)\left(X_{2}+\frac{1}{2}(d e Y-H)\right)^{2} \\
=\frac{1}{4}(d e Y-H)^{3}-\frac{1}{4}(d e Y)^{3} .
\end{gathered}
$$

Since $H \neq 0$, the quantities $d e Y$, de $Y-H$, and $(d e Y-H)^{3}-(d e Y)^{3}$ are all nonzero, so Lemma 2.3 may be applied. Thus, for fixed $d, e, H$, and $Y$, the values of $X_{1}$ and $X_{2}$ are determined up to $O\left(P^{\varepsilon}\right)$, and this fixes $X_{3}$ and $X_{4}$. For fixed $g, h$, and $\mathbf{u}$, this determines $\mathbf{v}$, and $y$ is then determined to $O(1)$ by a polynomial. Thus we have

$$
N_{6} \ll \sum_{d>P^{\gamma}} \sum_{1 \leq h^{\prime}, g^{\prime} \leq P / d} \sum_{x, \mathbf{u}} \sum_{H^{\prime}, e} \frac{h^{\prime} P^{1+\varepsilon}}{e} .
$$

For fixed $H^{\prime}$, a divisor estimate shows that there are $O\left(P^{\varepsilon}\right)$ possible choices for $e$, and for fixed $h$ and $x$ Lemma 2.1 shows that there are $O\left(P^{11 / 6+\varepsilon}\right)$ choices for $\mathbf{u}$. Thus on summing trivially over $g^{\prime}$ and $x$ we find that

$$
N_{6} \ll P^{1+\varepsilon} \sum_{d>P^{\gamma}} \frac{P}{d} \sum_{1 \leq h^{\prime} \leq P / d} h^{\prime} P \cdot P^{11 / 6+\varepsilon} \sum_{1 \leq H^{\prime} \leq 4 h^{\prime} P}\left(H^{\prime}\right)^{-1 / 3},
$$

on recalling that $e \gg\left|H^{\prime}\right|^{1 / 3}$. We now divide the sum over $H^{\prime}>h^{\prime}$ into dyadic intervals and consider the sum over $H^{\prime} \leq h^{\prime}$ separately. Since the choice of $h, g$, and $\mathbf{u}$ fixes the residue class of $H^{\prime}$ modulo $h^{\prime}$, there are $O\left(1+P / 2^{r}\right)$ choices for $H^{\prime}$ satisfying $4 h^{\prime} P / 2^{r+1}<H^{\prime} \leq 4 h^{\prime} P / 2^{r}$. Thus we have

$$
\sum_{1 \leq H^{\prime} \leq 4 h^{\prime} P}\left(H^{\prime}\right)^{-1 / 3} \ll\left(h^{\prime}\right)^{2 / 3}+\sum_{r=0}^{\infty} \frac{P}{2^{r}}\left(\frac{h^{\prime} P}{2^{r+1}}\right)^{-1 / 3} \ll\left(h^{\prime}\right)^{2 / 3}+\left(\frac{P^{2}}{h^{\prime}}\right)^{1 / 3},
$$

and here the second term is dominant whenever $h^{\prime} \leq P^{2 / 3}$, which certainly holds when $\gamma \geq 1 / 3$. Subject to this condition, we finally obtain

$$
\begin{aligned}
N_{6} & \ll P^{11 / 2+\varepsilon} \sum_{d>P^{\gamma}} d^{-1} \sum_{h^{\prime} \leq P / d}\left(h^{\prime}\right)^{2 / 3} \\
& \ll P^{43 / 6+\varepsilon} \sum_{d>P^{\gamma}} d^{-8 / 3} \ll P^{43 / 6-\frac{5}{3} \gamma+\varepsilon} .
\end{aligned}
$$

We now choose an optimal value of $\gamma \geq 1 / 3$. In order that $N_{5}$ and $N_{6}$ have the same order of magnitude, we set

$$
\frac{16}{3}-\frac{1}{3}(1+\beta)+\frac{4}{3} \gamma=\frac{43}{6}-\frac{5}{3} \gamma,
$$


which yields $\gamma=\frac{11}{18}+\frac{1}{9}(1+\beta)$. In view of our bound for $N_{3}$, we choose $\beta$ by setting

$$
\frac{35}{6}+\beta=\frac{43}{6}-\frac{5}{3}\left(\frac{11}{18}+\frac{1}{9}(1+\beta)\right),
$$

which gives $\beta=\frac{7}{64}$ and $\gamma=\frac{47}{64}$. The result of Theorem[1.2 now follows immediately on assembling the bounds for $N_{1}, \ldots, N_{6}$ and noting that $\frac{1}{6}-\frac{7}{64}=\frac{11}{192}$.

\section{Further Mean Value Estimates}

Here we use the result of the previous section to obtain estimates for higher moments, which will be required in our application of the Hardy-Littlewood method in Section 5. As usual, the sharpest estimates are obtained by considering solutions in which some of the variables have no large prime factors. Thus when $P$ and $R$ are positive integers, write

$$
\mathcal{A}(P, R)=\{n \in[1, P] \cap \mathbb{Z}: p \mid n, p \text { prime } \Longrightarrow p \leq R\}
$$

for the set of $R$-smooth numbers up to $P$, and define the exponential sum

$$
f(\boldsymbol{\alpha} ; P, R)=\sum_{x, y \in \mathcal{A}(P, R)} e\left(\alpha_{0} x^{3}+\alpha_{1} x^{2} y+\alpha_{2} x y^{2}+\alpha_{3} y^{3}\right) .
$$

It will also be useful to have some variables in a complete interval, so we define

$$
F(\boldsymbol{\alpha} ; P)=\sum_{1 \leq x, y \leq P} e\left(\alpha_{0} x^{3}+\alpha_{1} x^{2} y+\alpha_{2} x y^{2}+\alpha_{3} y^{3}\right)
$$

When there is no danger of confusion, we shall write $f(\boldsymbol{\alpha})=f(\boldsymbol{\alpha} ; P, R)$ and $F(\boldsymbol{\alpha})=$ $F(\boldsymbol{\alpha} ; P)$. Further, let

$$
S_{s, r}(P, R)=\int_{\mathbb{T}^{4}}|F(\boldsymbol{\alpha})|^{2 r}|f(\boldsymbol{\alpha})|^{2 s} d \boldsymbol{\alpha} .
$$

We adopt the convention that any statement involving $\varepsilon$ and $R$ means that for each $\varepsilon>0$ there exists $\eta=\eta(\varepsilon)>0$ such that the assertion holds whenever $R \leq P^{\eta}$. In this section, our implicit constants will depend at most on $\varepsilon$ unless otherwise noted. We start with an estimate for $S_{3,2}(P, R)$.

Lemma 3.1. One has

$$
S_{3,2}(P, R) \ll P^{12+\frac{1}{20}+\varepsilon} .
$$

Proof. Define the difference operator $\Delta_{1}^{*}$ by

$$
\Delta_{1}^{*}(f(x, y) ; h, g)=f(x+h, y+g)-f(x, y) .
$$

Then by Cauchy's inequality, one has

$$
\begin{aligned}
S_{3,2}(P, R) & =\int_{\mathbb{T}^{4}}\left|\sum_{x, h} \sum_{y, g} e\left(\sum_{i=0}^{3} \alpha_{i} \Delta_{1}^{*}\left(x^{3-i} y^{i} ; h, g\right)\right)\right|^{2}|f(\boldsymbol{\alpha})|^{6} d \boldsymbol{\alpha} \\
& \ll P^{2} \sum_{h, g} \int_{\mathbb{T}^{4}}\left|\sum_{x, y} e\left(\sum_{i=0}^{3} \alpha_{i} \Delta_{1}^{*}\left(x^{3-i} y^{i} ; h, g\right)\right)\right|^{2}|f(\boldsymbol{\alpha})|^{6} d \boldsymbol{\alpha},
\end{aligned}
$$

and hence

$$
S_{3,2}(P, R) \ll P^{2} U_{3,2}(P, R),
$$


where $U_{s, 2}(P, R)$ denotes the number of solutions of the system

$$
\begin{aligned}
3 h\left(x_{1}^{2}-x_{2}^{2}+h\left(x_{1}-x_{2}\right)\right) & =\sum_{i=1}^{s}\left(u_{i}^{3}-u_{s+i}^{3}\right), \\
h\left(2\left(x_{1} y_{1}-x_{2} y_{2}\right)+h\left(y_{1}-y_{2}\right)\right)+g\left(x_{1}^{2}-x_{2}^{2}+2 h\left(x_{1}-x_{2}\right)\right) & =\sum_{i=1}^{s}\left(u_{i}^{2} v_{i}-u_{s+i}^{2} v_{s+i}\right), \\
g\left(2\left(x_{1} y_{1}-x_{2} y_{2}\right)+g\left(x_{1}-x_{2}\right)\right)+h\left(y_{1}^{2}-y_{2}^{2}+2 g\left(y_{1}-y_{2}\right)\right) & =\sum_{i=1}^{s}\left(u_{i} v_{i}^{2}-u_{s+i} v_{s+i}^{2}\right), \\
3 g\left(y_{1}^{2}-y_{2}^{2}+g\left(y_{1}-y_{2}\right)\right) & =\sum_{i=1}^{s}\left(v_{i}^{3}-v_{s+i}^{3}\right),
\end{aligned}
$$

with

$$
1 \leq x_{i}, y_{i} \leq P, \quad u_{i}, v_{i} \in \mathcal{A}(P, R), \quad \text { and } \quad|h|,|g|<P .
$$

The argument is now very similar to the proof of Theorem 1.2 given in the previous section.

(i) Let $U_{1}$ denote the number of solutions counted by $U_{3,2}(P, R)$ for which

$$
h\left(x_{1}-x_{2}\right)\left(x_{1}+x_{2}+h\right)=g\left(y_{1}-y_{2}\right)\left(y_{1}+y_{2}+g\right)=0 .
$$

In this case, there are $O\left(P^{4}\right)$ choices for $h, g, \mathbf{x}$, and $\mathbf{y}$, and one sees that the number of choices for $\mathbf{u}$ and $\mathbf{v}$ is then

$$
\int_{\mathbb{T}^{4}}|f(\boldsymbol{\alpha})|^{6} d \boldsymbol{\alpha} \ll P^{6},
$$

on recalling Theorem 1.2 and considering the underlying Diophantine equations. Thus we have $U_{1} \ll P^{10}$.

(ii) Let $U_{2}$ denote the number of solutions counted by $U_{3,2}(P, R)$ for which exactly one of $h$ or $g$ is zero and (3.3) does not hold. First suppose that $h=0$ and $g \neq 0$. Then by Vaughan 13, Theorem 4.4, one has $O\left(P^{13 / 4+\varepsilon}\right)$ choices for $\mathbf{u}$, and by a trivial estimate there are $O\left(P^{3}\right)$ choices for $g$ and $\mathbf{y}$. Now for fixed $g$ and $\mathbf{y},[13$ ] again shows that there are $O\left(P^{13 / 4+\varepsilon}\right)$ choices for v. Finally, since $g\left(y_{1}-y_{2}\right) \neq 0$, $x_{1}$ and $x_{2}$ are determined to $O\left(P^{\varepsilon}\right)$ by the second and third equations above. The situation when $g=0$ and $h \neq 0$ is identical. Thus we see that $U_{2} \ll P^{19 / 2+\varepsilon}$.

(iii) Let $U_{3}$ denote the number of solutions counted by $U_{3,2}(P, R)$ for which $h g \neq 0$,

$$
\left(x_{1}-x_{2}\right)\left(y_{1}-y_{2}\right)\left(x_{1}+x_{2}+h\right)\left(y_{1}+y_{2}+g\right)=0 \text {, }
$$

and (3.3) does not hold. If $x_{1}=x_{2}$, then there are $O\left(P^{3}\right)$ choices for $h, g$, and $\mathbf{x}$ and $O\left(P^{13 / 4+\varepsilon}\right)$ choices for $\mathbf{u}$. Now, as in the previous section, we find that

$$
\begin{aligned}
\left(g u_{1}-h v_{1}\right)^{3}+ & \left(g u_{2}-h v_{2}\right)^{3}+\left(g u_{3}-h v_{3}\right)^{3} \\
& =\left(g u_{4}-h v_{4}\right)^{3}+\left(g u_{5}-h v_{5}\right)^{3}+\left(g u_{6}-h v_{6}\right)^{3},
\end{aligned}
$$

so by Hua 9], Theorem 4, there are $O\left(P^{7 / 2+\varepsilon}\right)$ choices for $\mathbf{v}$, and then $\mathbf{y}$ is determined to $O\left(P^{\varepsilon}\right)$ by a divisor estimate. The other cases are handled similarly, and thus $U_{3} \ll P^{39 / 4+\varepsilon}$. 
(iv) Write $d=(h, g)$, let $\beta$ be a parameter at our disposal, and let $U_{4}$ denote the number of solutions counted by $U_{3,2}(P, R)$ for which $0<|h g / d| \leq P^{5 / 4+\beta}$ and

$$
\left(x_{1}-x_{2}\right)\left(y_{1}-y_{2}\right)\left(x_{1}+x_{2}+h\right)\left(y_{1}+y_{2}+g\right) \neq 0 .
$$

In this case, there are $O\left(P^{5 / 4+\beta}\right)$ choices for the integer $h g / d$, whence by a divisor estimate there are $O\left(P^{5 / 4+\beta+\varepsilon}\right)$ choices for $h$ and $g$. Trivially, there are $O\left(P^{2}\right)$ choices for $\mathbf{x}$, and then we have $O\left(P^{13 / 4+\varepsilon}\right)$ choices for $\mathbf{u}$. Thus by applying Hua [9] to (3.4), we see that there are $O\left(P^{7 / 2+\varepsilon}\right)$ choices for $\mathbf{v}$, and then $\mathbf{y}$ is determined to $O\left(P^{\varepsilon}\right)$. Hence $U_{4} \ll P^{10+\beta+\varepsilon}$.

(v) Now let $\gamma$ be a parameter at our disposal, write $M=P^{5 / 4+\beta}$, and let $U_{5}$ be the number of solutions counted by $U_{3,2}(P, R)$ in which

$$
|h g / d|>M, \quad\left(x_{1}-x_{2}\right)\left(y_{1}-y_{2}\right)\left(x_{1}+x_{2}+h\right)\left(y_{1}+y_{2}+g\right) \neq 0,
$$

and $d=(h, g) \leq P^{\gamma}$. As before, we assume that $|h| \geq|g|$ and write $h^{\prime}=h / d$ and $g^{\prime}=g / d$, so that $\left(h^{\prime}, g^{\prime}\right)=1$. For any given $d$ and $\left|h^{\prime}\right| \geq\left|g^{\prime}\right|$, we divide both sides of (3.4) by $d^{3}$; then by Hua's Lemma there are $O\left(\left(\left|h^{\prime}\right| P\right)^{7 / 2+\varepsilon}\right)$ possible choices for $X_{1}, \ldots, X_{6}$, where $X_{i}=g u_{i}-h v_{i}$. With $X_{i}$ now fixed and $\left(h^{\prime}, g^{\prime}\right)=1$, any two choices for $u_{i}$ must be congruent modulo $h^{\prime}$, so one has $O\left(P /\left|h^{\prime}\right|\right)$ possibilities for each of $u_{1}, \ldots, u_{6}$, and this determines $\mathbf{v}$. Since $\mathbf{x}$ and $\mathbf{y}$ are then determined to $O\left(P^{\varepsilon}\right)$, we find that

$$
\begin{aligned}
U_{5} & \ll \sum_{d \leq P^{\gamma}} \sum_{1 \leq g \leq P / d} \sum_{h \geq \max (g, M / g d)}(h P)^{7 / 2+\varepsilon}(P / h)^{6} \\
& \ll P^{19 / 2+\varepsilon} \sum_{d \leq P^{\gamma}}\left(\sum_{g \leq(M / d)^{1 / 2}} \sum_{h \geq M / g d} h^{-5 / 2}+\sum_{g>(M / d)^{1 / 2}} \sum_{h \geq g} h^{-5 / 2}\right) \\
& \ll P^{19 / 2+\varepsilon} \sum_{d \leq P^{\gamma}}\left(\sum_{g \leq(M / d)^{1 / 2}}(M / g d)^{-3 / 2}+\sum_{g>(M / d)^{1 / 2}} g^{-3 / 2}\right) \\
& \ll P^{19 / 2+\varepsilon} \sum_{d \leq P^{\gamma}}\left((M / d)^{-3 / 2}(M / d)^{5 / 4}+(M / d)^{-1 / 4}\right) \\
& \ll P^{19 / 2+\varepsilon} M^{-1 / 4} \sum_{d \leq P^{\gamma}} d^{1 / 4} \ll P^{\frac{19}{2}-\frac{1}{4}\left(\frac{5}{4}+\beta\right)+\frac{5}{4} \gamma+\varepsilon} .
\end{aligned}
$$

(vi) Finally, let $U_{6}$ be the number of solutions counted by $U_{3,2}(P, R)$ with (3.5) and $d>P^{\gamma}$. For fixed $d$, there are at most $(P / d)^{2}$ choices for $h$ and $g$ and $P^{2}$ choices for $\mathbf{x}$. Now, for given $h$ and $\mathbf{x}$, Theorem 4.4 of Vaughan [13] shows that there are $O\left(P^{13 / 4+\varepsilon}\right)$ choices for $\mathbf{u}$, and then on recalling (3.4) and Hua 9], Theorem 4, we see that there are $O\left(P^{7 / 2+\varepsilon}\right)$ choices for $\mathbf{v}$, from which $\mathbf{y}$ is determined to $O\left(P^{\varepsilon}\right)$. Thus we have

$$
U_{6} \ll P^{43 / 4+\varepsilon} \sum_{d>P^{\gamma}} d^{-2} \ll P^{43 / 4-\gamma+\varepsilon} .
$$

To optimize the results of (v) and (vi), we set

$$
9+\frac{3}{16}-\frac{1}{4} \beta+\frac{5}{4} \gamma=10+\frac{3}{4}-\gamma
$$


which gives $\gamma=\frac{25}{36}+\frac{1}{9} \beta$. Now on recalling (iv), we choose $\beta$ so that

$$
10+\beta=\frac{43}{4}-\frac{25}{36}-\frac{1}{9} \beta
$$

which gives $\beta=\frac{1}{20}$. Hence we have $U_{3,2}(P, R) \ll P^{10+\frac{1}{20}}$, and the lemma follows on recalling (3.1).

Before proceeding, we record an easy consequence of Lemma 3.1

Lemma 3.2. One has

$$
S_{2,2}(P, R) \ll P^{9+\frac{1}{40}+\varepsilon} .
$$

Proof. By Theorem 1.2, we have

$$
\int_{\mathbb{T}^{4}}|F(\boldsymbol{\alpha})|^{6} d \boldsymbol{\alpha} \ll P^{6}
$$

Thus by applying the Cauchy-Schwarz inequality and Lemma 3.1] we obtain

$$
S_{2,2}(P, R) \ll\left(\int_{\mathbb{T}^{4}}|F(\boldsymbol{\alpha})|^{6} d \boldsymbol{\alpha}\right)^{1 / 2}\left(\int_{\mathbb{T}^{4}}|F(\boldsymbol{\alpha})|^{2}|f(\boldsymbol{\alpha})|^{8} d \boldsymbol{\alpha}\right)^{1 / 2} \ll P^{9+\frac{1}{40}+\varepsilon}
$$

on considering the underlying Diophantine equations.

We now proceed to estimate some higher moments.

Lemma 3.3. One has

$$
S_{4,2}(P, R) \ll P^{15+\frac{1}{3}+\varepsilon} .
$$

Proof. By Cauchy's inequality, we have $S_{4,2}(P, R) \ll P^{2} U_{4,2}(P, R)$, and the estimation of $U_{4,2}(P, R)$ proceeds almost exactly as in the proof of Lemma 3.1. The only modifications are that we use Lemma 3.2 in place of Theorem 1.2 in the analysis of case (i), and we replace the 6th moment estimates of $P^{13 / 4+\varepsilon}$ and $P^{7 / 2+\varepsilon}$ by Hua's 8th moment estimate of $P^{5+\varepsilon}$. Taking $M=P^{4 / 3}$ and $\gamma=2 / 3$ produces identical bounds for the final three cases, and hence gives an optimal result.

We remark that the argument of the preceding proof in fact shows that one may replace $S_{4,2}(P, R)$ by $S_{2,4}(P, R)$ in the statement of Lemma 3.3 Further, we note that tiny improvements in the exponents of Lemmata 3.1 and 3.2 may be achieved by using the results of Wooley [17, [20] in place of Vaughan [13], but such improvements do not have significant consequences in the present application.

For higher moments, we apply the (single) efficient differencing procedure of [10. Although our methods always allow us to take a few variables ranging over a complete interval, we will often simplify by stating results for mean values in which all the variables are smooth. Thus we adopt the notation of writing $S_{s}(P, R)$ for $S_{s, 0}(P, R)$. Further, we say that $\Delta_{s}$ is an admissible exponent if one has $S_{s}(P, R) \ll$ $P^{\lambda_{s}+\varepsilon}$, where $\lambda_{s}=4 s-12+\Delta_{s}$, and in this situation we call $\lambda_{s}$ a permissible exponent. To this point we have obtained the admissible exponents

$$
\Delta_{3}=6, \quad \Delta_{4}=5 \frac{1}{40}, \quad \Delta_{5}=4 \frac{1}{20}, \quad \text { and } \quad \Delta_{6}=3 \frac{1}{3} .
$$

The above method of generating admissible exponents becomes noticeably less effective when $s>6$, since the maximum savings of $P^{3}$ in estimating the number of solutions of

$$
u_{1}^{3}+\cdots+u_{t}^{3}=u_{t+1}^{3}+\cdots+u_{2 t}^{3}
$$


is already achieved when $t=4$. Since the results of [10 are directly applicable only for $s \geq 11$, we will need the following lemma to work out admissible exponents when $s$ lies in the intermediate range.

Lemma 3.4. One has

$$
S_{s+2}(P, R) \ll P^{5} S_{s}(P, R)+P^{19 / 6+\varepsilon} S_{s-1,2}(P, R)+P^{\frac{2}{3} s+6+\varepsilon} S_{s}\left(P^{5 / 6}, R\right) .
$$

Proof. This follows on using [10, Lemma 3.2, in the initial stages of the argument of [10], Lemma 5.1.

We now apply Lemma 3.4 repeatedly to obtain admissible exponents $\Delta_{s}$ for $7 \leq s \leq 12$. First of all, by using Lemma 3.3 and making a trivial estimate, we see that

$$
S_{5,2}(P, R) \ll P^{19+\frac{1}{3}+\varepsilon},
$$

and using this together with Lemma 3.3 in Lemma 3.4 gives

$$
S_{8}(P, R) \ll P^{22+\frac{7}{9}+\varepsilon} .
$$

Then on using the Cauchy-Schwarz inequality to interpolate between $S_{4,2}$ and $S_{8}$, we obtain

$$
S_{7}(P, R) \ll\left(S_{4,2}(P, R)\right)^{1 / 2}\left(S_{8}(P, R)\right)^{1 / 2} \ll P^{19+\frac{1}{18}+\varepsilon} .
$$

Putting (3.7) and (3.8) into Lemma 3.4 now yields

$$
S_{9}(P, R) \ll P^{26+\frac{59}{108}+\varepsilon} \quad \text { and } \quad S_{10}(P, R) \ll P^{30+\frac{17}{54}+\varepsilon},
$$

on recalling Lemma 3.3 and noting the trivial inequality

$$
S_{t, 2}(P, R) \leq P^{8} S_{t}(P, R)
$$

Continuing the iteration, we find that

$$
S_{11}(P, R) \ll P^{34+\frac{79}{648}+\varepsilon} \text { and } S_{12}(P, R) \ll P^{37+\frac{301}{324}+\varepsilon},
$$

and thus we have the admissible exponents

$$
\begin{array}{rlr}
\Delta_{7}=3 \frac{1}{18}, \quad \Delta_{8}=2 \frac{7}{9}, & \Delta_{9}=2 \frac{59}{108}, \\
\Delta_{10}=2 \frac{17}{54}, & \Delta_{11}=2 \frac{79}{648}, & \Delta_{12}=\frac{625}{324} .
\end{array}
$$

Further admissible exponents can now be read off from Lemma 5.1 of [10]. Namely, if $s \geq 11$ and $\Delta_{s}$ is admissible, then the exponent

$$
\Delta_{s+2 t}=\Delta_{s}(5 / 6)^{t}
$$

is also admissible.

\section{WeYl Differencing}

Here we obtain estimates for the modulus of the exponential sum $F(\boldsymbol{\alpha})$ when at least one of the $\alpha_{j}$ is badly approximated by rationals. In [10], estimates of this type were obtained for $f(\boldsymbol{\alpha})$ by using the large sieve to relate the modulus of the sum to known mean values. This treatment allowed us to obtain bounds of the form $P^{2-\sigma(k)+\varepsilon}$, where $\sigma(k)^{-1} \asymp k^{3} \log k$, and for large $k$ this is substantially better than the exponential decay that results from Weyl differencing. For $k=3$, however, we 
are much better off applying a two-fold Weyl differencing procedure. For purposes of application, it is useful to consider the slightly more general exponential sum

$$
F(\boldsymbol{\alpha} ; P, Q)=\sum_{1 \leq x \leq P} \sum_{1 \leq y \leq Q} e\left(\alpha_{0} x^{3}+\alpha_{1} x^{2} y+\alpha_{2} x y^{2}+\alpha_{3} y^{3}\right) .
$$

Lemma 4.1. Suppose that $Q \asymp P$ and that for some $j$ we have $q_{j} \in \mathbb{N}$ and $a_{j} \in \mathbb{Z}$ with

$$
\left(q_{j}, a_{j}\right)=1 \quad \text { and } \quad\left|q_{j} \alpha_{j}-a_{j}\right| \leq q_{j}^{-1} .
$$

Then one has

$$
|F(\boldsymbol{\alpha} ; P, Q)| \ll P^{2+\varepsilon}\left(q_{j}^{-1}+P^{-1}+q_{j} P^{-3}\right)^{1 / 4} .
$$

Proof. First suppose that (4.1) holds with $j=0$. Then by Weyl's inequality (see for instance Lemma 2.4 of Vaughan [14]) one has

$$
\begin{aligned}
|F(\boldsymbol{\alpha} ; P, Q)| & \leq \sum_{y \leq Q}\left|\sum_{x \leq P} e\left(\alpha_{0} x^{3}+\alpha_{1} x^{2} y+\alpha_{2} x y^{2}\right)\right| \\
& \ll Q P^{1+\varepsilon}\left(q_{0}^{-1}+P^{-1}+q_{0} P^{-3}\right)^{1 / 4},
\end{aligned}
$$

and the result follows. Note that if instead (4.1) holds with $j=3$, then we obtain the same conclusion simply by interchanging the roles of $x$ and $y$ in the above argument.

Now suppose that (4.1) holds with $j=1$. Then by Cauchy's inequality we have

$$
\begin{aligned}
|F(\boldsymbol{\alpha})|^{2} & \leq Q \sum_{y \leq Q}\left|\sum_{x \leq P} e\left(\alpha_{0} x^{3}+\alpha_{1} x^{2} y+\alpha_{2} x y^{2}\right)\right|^{2} \\
& \ll P \sum_{y \leq Q}\left(P+\sum_{x, h} e\left(\alpha_{0}\left(3 x^{2} h+3 x h^{2}+h^{3}\right)+\alpha_{1} y\left(2 x h+h^{2}\right)+\alpha_{2} y^{2} h\right)\right) \\
& \ll P^{3}+P \sum_{y \leq Q} \sum_{x, h} e\left(\alpha_{0}\left(3 x^{2} h+3 x h^{2}+h^{3}\right)+\alpha_{1} y\left(2 x h+h^{2}\right)+\alpha_{2} y^{2} h\right),
\end{aligned}
$$

where the second sum is over $x$ and $h \neq 0$ with $1 \leq x \leq P$ and $1-x \leq h \leq P-x$, and where we have abbreviated $F(\boldsymbol{\alpha} ; P, Q)$ by $\bar{F}(\boldsymbol{\alpha})$. Then on using Cauchy's inequality again we obtain

$$
\begin{aligned}
|F(\boldsymbol{\alpha})|^{4} & \ll P^{6}+P^{4} \sum_{x, h}\left|\sum_{y \leq Q} e\left(\alpha_{0}\left(3 x^{2} h+3 x h^{2}+h^{3}\right)+\alpha_{1} y\left(2 x h+h^{2}\right)+\alpha_{2} y^{2} h\right)\right|^{2} \\
& \ll P^{6}+P^{4} \sum_{x, h}\left(P+\sum_{y, g} e\left(\alpha_{1} g\left(2 x h+h^{2}\right)+\alpha_{2} h\left(2 y g+g^{2}\right)\right)\right) \\
& \ll P^{7}+P^{4} \sum_{x, h, y, g} e\left(\alpha_{1} g\left(2 x h+h^{2}\right)+\alpha_{2} h\left(2 y g+g^{2}\right)\right) \\
& \ll P^{7}+P^{4} \sum_{1 \leq|h|,|g| \leq P} \sum_{y \in I(Q, g)}\left|\sum_{x \in I(P, h)} e\left(2 \alpha_{1} g h x\right)\right|
\end{aligned}
$$


where $I(P, h)=[1, P] \cap[1-h, P-h]$. Thus on summing the geometric progression, recalling a standard divisor function estimate, and using Lemma 2.2 of [14], we find that

$$
\begin{aligned}
|F(\boldsymbol{\alpha} ; P, Q)|^{4} & \ll P^{7}+Q P^{4} \sum_{1 \leq|h|,|g| \leq P} \min \left(P,\left\|2 \alpha_{1} g h\right\|^{-1}\right) \\
& \ll P^{7}+Q P^{4+\varepsilon} \sum_{n \leq 2 P^{2}} \min \left(P,\left\|\alpha_{1} n\right\|^{-1}\right) \\
& \ll P^{7}+Q P^{7+\varepsilon}\left(q_{1}^{-1}+P^{-1}+q_{1} P^{-3}\right),
\end{aligned}
$$

whence

$$
|F(\boldsymbol{\alpha} ; P, Q)| \ll P^{2+\varepsilon}\left(q_{1}^{-1}+P^{-1}+q_{1} P^{-3}\right)^{1 / 4} .
$$

Again, the same conclusion follows when (4.1) holds with $j=2$ by repeating the argument with the roles of $x$ and $y$ reversed.

Next we record a consequence of the above lemma, which will be useful in our application of the circle method.

Lemma 4.2. Let $\alpha_{0}, \ldots, \alpha_{3}$ be real numbers with the property that whenever there exist $q \in \mathbb{N}$ and $a_{0}, \ldots, a_{3} \in \mathbb{Z}$ with $\left(q, a_{0}, \ldots, a_{3}\right)=1$ and $\left|q \alpha_{j}-a_{j}\right| \leq P^{\delta-3}$ one has $q>P^{\delta}$. Then whenever $Q \asymp P$ one has

$$
|F(\boldsymbol{\alpha} ; P, Q)| \ll P^{2-\delta / 16+\varepsilon} .
$$

Proof. Let $\alpha_{0}, \ldots, \alpha_{3}$ be as in the statement of the lemma, and write $\nu=\delta / 4$. For each $j$, Dirichlet's Theorem allows us to find $q_{j} \in \mathbb{N}$ and $b_{j} \in \mathbb{Z}$ with $\left(q_{j}, b_{j}\right)=1$ such that $\left|q_{j} \alpha_{j}-b_{j}\right| \leq P^{\nu-3}$ and $q_{j} \leq P^{3-\nu}$. If $q_{j}>P^{\nu}$ for some $j$, then the conclusion follows from Lemma 4.1 Otherwise, put $q=\left[q_{0}, \ldots, q_{3}\right]$ and $a_{j}=b_{j} q / q_{j}$. Then we have $\left(q, a_{0}, \ldots, a_{3}\right)=1$ and $q \leq q_{j} P^{3 \nu} \leq P^{\delta}$ for each $j$, and hence

$$
\left|\alpha_{j}-a_{j} / q\right| \leq q_{j}^{-1} P^{\nu-3} \leq q^{-1} P^{\delta-3} \quad(0 \leq j \leq 3),
$$

contradicting the hypothesis of the lemma.

\section{The Circle Method}

Now we are in a position to prove Theorem 1.1 by applying the circle method along the lines of [10], $\S 10$. The following lemma provides us with non-singular local solutions to (1.1).

Lemma 5.1. If $s \geq 14$ and $c_{1}, \ldots, c_{s}$ are non-zero integers, then the system (1.1) has a non-singular real solution and a non-singular $p$-adic solution for all primes $p$.

Proof. After setting

$$
y_{1}=\cdots=y_{7}=0, \quad x_{8}=\cdots=x_{14}=0, \quad \text { and } \quad x_{i}=y_{i}=0 \quad(i>14),
$$

the system (1.1) reduces to

$$
c_{1} x_{1}^{3}+\cdots+c_{7} x_{7}^{3}=0 \text { and } c_{8} y_{8}^{3}+\cdots+c_{14} y_{14}^{3}=0
$$

By a well-known result of Baker 2], each of these equations has a non-trivial integral solution; suppose that $\mathbf{x}$ and $\mathbf{y}$ are solutions with $x_{I}$ and $y_{J}$ non-zero. Then on writing

$$
\psi_{j}(\mathbf{x}, \mathbf{y})=c_{1} x_{1}^{3-j} y_{1}^{j}+\cdots+c_{s} x_{s}^{3-j} y_{s}^{j} \quad(0 \leq j \leq 3)
$$


we have

$$
\operatorname{det}\left(\frac{\partial \psi_{j}}{\partial x_{I}}, \frac{\partial \psi_{j}}{\partial y_{I}}, \frac{\partial \psi_{j}}{\partial x_{J}}, \frac{\partial \psi_{j}}{\partial y_{J}}\right)_{0 \leq j \leq 3}=\left(3 c_{I} c_{J}\right)^{2} x_{I}^{4} y_{J}^{4} \neq 0 .
$$

Thus $(\mathbf{x}, \mathbf{y})$ is a non-singular integer solution of (1.1), so it is non-singular in each local field as well.

By Lemma 5.1] and [10], Lemma 10.2, we may assume that the system (1.1) has a non-singular real solution $(\boldsymbol{\eta}, \boldsymbol{\xi})$ with $0<\left|\eta_{i}\right|,\left|\xi_{i}\right|<\frac{1}{2}$ for $i=1, \ldots, s$. For each $i$, we write

$$
\eta_{i}^{+}=\eta_{i}+\frac{1}{2}\left|\eta_{i}\right| \quad \text { and } \quad \eta_{i}^{-}=\eta_{i}-\frac{1}{2}\left|\eta_{i}\right|
$$

and

$$
\xi_{i}^{+}=\xi_{i}+\frac{1}{2}\left|\xi_{i}\right| \quad \text { and } \quad \xi_{i}^{-}=\xi_{i}-\frac{1}{2}\left|\xi_{i}\right| .
$$

Now let $P$ be a large positive number, put $R=P^{\eta}$ with $\eta \leq \eta_{0}(\varepsilon)$, and let $c_{1}, \ldots, c_{s}$ be non-zero integers. Throughout this section, the implicit constants arising in our analysis may depend on $c_{1}, \ldots, c_{s}$ and on the real solution $(\boldsymbol{\eta}, \boldsymbol{\xi})$. We define the exponential sums

$$
F_{i}(\boldsymbol{\alpha})=\sum_{\eta_{i}^{-} P<x \leq \eta_{i}^{+} P} \sum_{\xi_{i}^{-} P<y \leq \xi_{i}^{+} P} e\left(c_{i}\left(\alpha_{0} x^{3}+\alpha_{1} x^{2} y+\alpha_{2} x y^{2}+\alpha_{3} y^{3}\right)\right)
$$

and

$$
f_{i}(\boldsymbol{\alpha})=\sum_{\substack{\eta_{i}^{-} P<x \leq \eta_{i}^{+} P \\|x| \in \mathcal{A}(P, R)}} \sum_{\substack{\xi_{i}^{-} P<y \leq \xi_{i}^{+} P \\|y| \in \mathcal{A}(P, R)}} e\left(c_{i}\left(\alpha_{0} x^{3}+\alpha_{1} x^{2} y+\alpha_{2} x y^{2}+\alpha_{3} y^{3}\right)\right) .
$$

Further, write $s=t+2 u+v$ and define

$$
\mathcal{F}(\boldsymbol{\alpha})=\prod_{i=1}^{t} F_{i}(\boldsymbol{\alpha}) \quad \text { and } \quad \mathcal{G}(\boldsymbol{\alpha})=\prod_{i=t+1}^{s} f_{i}(\boldsymbol{\alpha}) .
$$

Finally, let

$$
R_{s}(P)=\int_{\mathbb{T}^{4}} \mathcal{F}(\boldsymbol{\alpha}) \mathcal{G}(\boldsymbol{\alpha}) d \boldsymbol{\alpha},
$$

and observe that $R_{s}(P)$ is a lower bound for the number of integral solutions of (1.1) lying in the box $[-P, P]^{2 s}$.

We dissect $\mathbb{T}^{4}$ into major and minor arcs as follows. Write $c=\max \left|c_{i}\right|$, let $\delta \in(0,1)$ be a parameter at our disposal, define

$$
\mathfrak{M}=\underset{\substack{1 \leq a_{0}, \ldots, a_{3} \leq q \leq c P^{\delta} \\\left(q, a_{0}, \ldots, a_{3}\right)=1}}{\bigcup} \mathfrak{M}(q, \mathbf{a}),
$$

where

$$
\mathfrak{M}(q, \mathbf{a})=\left\{\boldsymbol{\alpha} \in \mathbb{T}^{4}:\left|q \alpha_{i}-a_{i}\right| \leq P^{\delta-3}(0 \leq i \leq 3)\right\},
$$

and put $\mathfrak{m}=\mathbb{T}^{4} \backslash \mathfrak{M}$. It is easily seen that the $\mathfrak{M}(q, \mathbf{a})$ are disjoint whenever $P$ is sufficiently large and $\delta<1$. 
We further define the pruned major arcs by

$$
\mathfrak{N}=\bigcup_{\substack{1 \leq a_{0}, \ldots, a_{3} \leq q \leq W \\\left(q, a_{0}, \ldots, a_{3}\right)=1}} \mathfrak{N}(q, \mathbf{a}),
$$

where $W<c P^{\delta}$ is a parameter at our disposal and

$$
\mathfrak{N}(q, \mathbf{a})=\left\{\boldsymbol{\alpha} \in \mathbb{T}^{4}:\left|\alpha_{i}-a_{i} / q\right| \leq W P^{-3}(0 \leq i \leq 3)\right\} .
$$

The following pruning lemma is reminiscent of [16], Lemma 9.2.

Lemma 5.2. If $t \geq 16$ and $\Delta_{u}<(1-\delta) t$ is an admissible exponent, then one has

$$
\int_{\mathfrak{M} \backslash \mathfrak{N}}\left|F_{i}(\boldsymbol{\alpha})\right|^{t}\left|f_{j}(\boldsymbol{\alpha})\right|^{2 u} d \boldsymbol{\alpha} \ll W^{-\sigma} P^{2 t+4 u-12}
$$

for some $\sigma>0$.

Proof. When $\boldsymbol{\alpha} \in \mathfrak{M}(q, \mathbf{a})$, we have by a simple modification of [10], Lemma 8.1, that

$$
F_{i}(\boldsymbol{\alpha})=V_{i}(\boldsymbol{\alpha})+O\left(P^{1+\delta}\right)
$$

where

$$
\begin{gathered}
V_{i}(\boldsymbol{\alpha})=q^{-2} S_{i}(q, \mathbf{a}) v_{i}(\boldsymbol{\alpha}-\mathbf{a} / q), \\
S_{i}(q, \mathbf{a})=\sum_{1 \leq x, y \leq q} e\left(\frac{c_{i}\left(a_{0} x^{3}+a_{1} x^{2} y+a_{2} x y^{2}+a_{3} y^{3}\right)}{q}\right),
\end{gathered}
$$

and

$$
v_{i}(\boldsymbol{\beta})=\int_{\eta_{i}^{-} P}^{\eta_{i}^{+} P} \int_{\xi_{i}^{-} P}^{\xi_{i}^{+} P} e\left(c_{i}\left(\beta_{0} \gamma^{3}+\beta_{1} \gamma^{2} \nu+\beta_{2} \gamma \nu^{2}+\beta_{3} \nu^{3}\right)\right) d \gamma d \nu .
$$

Then whenever $\mathcal{M} \subset \mathfrak{M}$, we have

$$
\int_{\mathcal{M}}\left|F_{i}(\boldsymbol{\alpha})\right|^{t}\left|f_{j}(\boldsymbol{\alpha})\right|^{2 u} d \boldsymbol{\alpha} \ll P^{4 u} \int_{\mathcal{M}}\left|V_{i}(\boldsymbol{\alpha})\right|^{t} d \boldsymbol{\alpha}+P^{t(1+\delta)} \int_{\mathbb{T}^{4}}\left|f_{j}(\boldsymbol{\alpha})\right|^{2 u} d \boldsymbol{\alpha} .
$$

On using the estimates

$$
S_{i}(q, \mathbf{a}) \ll q^{2-1 / 3+\varepsilon}
$$

and

$$
v_{i}(\boldsymbol{\beta}) \ll P^{2} \prod_{i=0}^{3}\left(1+P^{3}\left|\beta_{i}\right|\right)^{-1 / 12}
$$

(see for example [1]), we find that whenever $t \geq 16$ one has

$$
\int_{\mathfrak{M} \backslash \mathfrak{N}}\left|V_{i}(\boldsymbol{\alpha})\right|^{t} d \boldsymbol{\alpha} \ll P^{2 t-12} W^{-1 / 3} .
$$

Moreover, it is clear by considering the underlying Diophantine equations that all the mean value estimates from Section 3 hold with $f(\boldsymbol{\alpha})$ replaced by $f_{j}(\boldsymbol{\alpha})$, so we have

$$
\int_{\mathbb{T}^{4}}\left|f_{j}(\boldsymbol{\alpha})\right|^{2 u} d \boldsymbol{\alpha} \ll P^{4 u-12+\Delta_{u}+\varepsilon} \ll P^{4 u-12+t(1-\delta)-\gamma}
$$

for some $\gamma>0$, and the lemma follows. 
We are now ready to derive the lower bound for $R_{s}(P)$. If $\boldsymbol{\alpha} \in \mathfrak{m}$, then by the argument of [10], Lemma 10.3, one sees that the hypotheses of Lemma 4.2 hold with $\boldsymbol{\alpha}$ replaced by $c_{i} \boldsymbol{\alpha}$, and hence

$$
\sup _{\boldsymbol{\alpha} \in \mathfrak{m}}\left|F_{i}(\boldsymbol{\alpha})\right| \ll P^{2-\delta / 16+\varepsilon} .
$$

Thus by Hölder's inequality we have

$$
\begin{aligned}
\int_{\mathfrak{m}}|\mathcal{F}(\boldsymbol{\alpha}) \mathcal{G}(\boldsymbol{\alpha})| d \boldsymbol{\alpha} & \ll P^{2 t+2 v-\frac{t \delta}{16}+\varepsilon} \prod_{i=t+1}^{t+2 u}\left(\int_{\mathbb{T}^{4}}\left|f_{i}(\boldsymbol{\alpha})\right|^{2 u} d \boldsymbol{\alpha}\right)^{1 / 2 u} \\
& \ll P^{2 s-12+\Delta_{u}-\frac{t \delta}{16}+\varepsilon} \ll P^{2 s-12-\tau}
\end{aligned}
$$

for some $\tau>0$, provided that

$$
t \delta>16 \Delta_{u} .
$$

In view of the condition in Lemma 5.2 the optimal choice for $\delta$ satisfies $1-\delta=\frac{\delta}{16}$, so we take $\delta=16 / 17$. Now on recalling (3.9) and (3.10) we see that the exponent

$$
\Delta_{16}=\frac{625}{324}\left(\frac{5}{6}\right)^{2}<\frac{23}{17}
$$

is admissible, and it transpires that choosing $t=23$ and $u=16$ minimizes the quantity $t+2 u$ subject to (5.1). Thus on taking $v \geq 2$, we have a total of $s \geq 57$ variables.

It now suffices to deal with the major arcs. By applying Lemma 5.2 and making a trivial estimate, we have for some $i$ and $j$ that

$$
\int_{\mathfrak{M} \backslash \mathfrak{N}}|\mathcal{F}(\boldsymbol{\alpha}) \mathcal{G}(\boldsymbol{\alpha})| d \boldsymbol{\alpha} \ll P^{2 v} \int_{\mathfrak{M} \backslash \mathfrak{N}}\left|F_{i}(\boldsymbol{\alpha})\right|^{23}\left|f_{j}(\boldsymbol{\alpha})\right|^{32} d \boldsymbol{\alpha} \ll P^{2 s-12} W^{-\sigma},
$$

and so it suffices to deal with the pruned major arc $\mathfrak{N}$. But it follows immediately from the analysis of [10], $§ 10$, that

$$
\int_{\mathfrak{N}} \mathcal{F}(\boldsymbol{\alpha}) \mathcal{G}(\boldsymbol{\alpha}) d \boldsymbol{\alpha} \gg P^{2 s-12}
$$

provided that $W$ is taken to be a suitably small power of $\log P$.

Finally, we need to relate $R_{s}(P)$ to $L_{s}(P)$ by accounting for the possibility that distinct solutions of (1.1) correspond to different parameterizations of the same line. By the argument of [10], Lemma 10.12, we have

$$
R_{s}(P, d) \ll \frac{P^{2 v}}{d^{v}} \int_{\mathbb{T}^{4}}|\mathcal{F}(\boldsymbol{\alpha})|\left(\prod_{i=24}^{55}\left|f_{i}(\boldsymbol{\alpha})\right|\right) d \boldsymbol{\alpha} \ll \frac{P^{2 s-12}}{d^{2}},
$$

where $R_{s}(P, d)$ denotes the number of solutions of (1.1) counted by $R_{s}(P)$ for which $\operatorname{gcd}\left(x_{1}, \ldots, x_{s}\right)=d$. Thus on following through the corresponding argument in [10], we find that $L_{s}(P) \gg P^{2 s-12}$, and this completes the proof of Theorem 1.1.

As mentioned in the introduction, essentially the same analysis may be applied to establish a result on the two-dimensional version of Waring's problem discussed in [10]. In that argument, we may clearly take $v=0$, and it follows immediately that $G_{1}^{*}(3) \leq 55$. 


\section{REFERENCES}

1. G. I. Arkhipov, A. A. Karatsuba, and V. N. Chubarikov, Multiple trigonometric sums, Trudy Mat. Inst. Steklov. 151 (1980); English Transl., Proc. Steklov Inst. Math. 1982, no. 2 (151). MR 82i: 10045

2. R. C. Baker, Diagonal cubic equations II, Acta Arith. 53 (1989), 217-250. MR 91b:11100a

3. B. J. Birch, Homogeneous forms of odd degree in a large number of variables, Mathematika 4 (1957), 102-105. MR 20:3828

4. R. Brauer, A note on systems of homogeneous algebraic equations, Bull. Amer. Math. Soc. 51 (1945), 749-755. MR 7:108i

5. T. Estermann, Einige Sätze über quadratfreie Zahlen, Math. Ann. 105 (1931), 653-662.

6. D. R. Heath-Brown, The density of rational points on cubic surfaces, Acta Arith. 79 (1997), 17-30. MR 98h:11083

7. C. Hooley, On the representations of a number as the sum of four cubes, Proc. London Math. Soc. (3) 36 (1978), 117-140. MR 58:21932a

8. - On the numbers that are representable as the sum of two cubes, J. Reine Angew. Math. 314 (1980), 146-173. MR 81d:10036

9. L.-K. Hua, Additive theory of prime numbers, A.M.S., Providence, RI, 1965. MR 32:2614

10. S. T. Parsell, Multiple exponential sums over smooth numbers J. Reine Angew. Math (to appear).

11. W. M. Schmidt, On cubic polynomials III. Systems of p-adic equations, Monatsh. Math. 93 (1982), 211-223. MR 83m:10062

12. - On cubic polynomials IV. Systems of rational equations, Monatsh. Math. 93 (1982), 329-348. MR 83m:10063

13. R. C. Vaughan, A new iterative method in Waring's problem, Acta Math. 162 (1989), 1-71. MR 90c: 11072

14. 1997. MR 98a:11133

15. R. C. Vaughan and T. D. Wooley, Further improvements in Waring's problem, Acta Math. 174 (1995), 147-240. MR 96j:11129

16. T. D. Wooley, On simultaneous additive equations II, J. Reine Angew. Math. 429 (1991), 141-198. MR 92e:11025b

17. _ Breaking classical convexity in Waring's problem: Sums of cubes and quasi-diagonal behaviour, Invent. Math. 122 (1995), 421-451. MR 97d:11148

18. _ Sums of two cubes, Internat. Math. Res. Notices 4 (1995), 181-185. MR 96a:11103

19. Linear spaces on cubic hypersurfaces and pairs of homogeneous cubic equations, Bull. London Math. Soc. 29 (1997), 556-562. MR 99e:11036

20 . Sums of three cubes, Mathematika (to appear).

Department of Mathematics, University of Michigan, 525 East University Avenue, Ann Arbor, Michigan 48109-1109

Current address: Department of Mathematics, Texas A\&M University, College Station, Texas 77843-3368

E-mail address: parsell@alum.mit.edu 